\title{
Pimavanserin could be useful for treating psychosis in Parkinson disease
}

The selective serotonin 5-HT2A inverse agonist pimavanserin has shown promise for the treatment of psychosis in Parkinson disease (PD) in a phase III trial. "The findings could help to address a massive unmet need in PD," says Clive Ballard, who led the study.

Psychosis affects more than $50 \%$ of patients with $\mathrm{PD}$. It usually involves hallucinations and delusions, which can be distressing for patients and caregivers. Typical antipsychotics are unsuitable for use in patients with PD as they can cause dopamine D2 antagonism, thereby worsening motor symptoms. "Clozapine is approved as a second-line treatment but is associated with a number of blackbox safety warnings and requires stringent blood monitoring," says Ballard.

The new study comprised a 6-week, randomized, double-blind, placebocontrolled trial in 199 patients with moderate to severe PD who had been experiencing psychotic symptoms for at least 3 years. Prior to treatment, participants entered a 2-week lead-in phase involving psychosocial therapy adapted for PD. "The aim of the lead-in phase was to reduce the large placebo response that hampered a previous trial of pimavanserin for PD psychosis," explains Ballard. Participants were then randomly assigned to receive $40 \mathrm{mg}$ per day pimavanserin or placebo.

\section{The findings could help to address a massive unmet need in PD... 77}

Patients were assessed at baseline and days 15, 29 and 43 using the PD-adapted scale for assessment of positive symptoms (SAPS-PD), which rates seven individual symptoms, as well as global hallucinations and global delusions. Pimavanserin treatment was associated with a decrease of 5.79 points in mean SAPS-PD score, compared with a 2.79-point decrease in the placebo group $(P=0.001)$. This finding was supported by secondary outcome measures, such as improvement on the clinical global impression scale and the caregiver burden scale, and the treatment was well-tolerated overall.

"A strength of the study is that outcomes were independently assesssedby raters, investigators and carers-which gives us much more confidence in the results," concludes Ballard. He says that licensing applications will go ahead in Europe and the USA on the basis of the study results. A trial of pimavanserin for the treatment of psychosis in Alzheimer disease is also planned.

Katie Kingwell

Original article Cummings, J. et al. Pimavanserin for patients with Parkinson's disease psychosis: a randomised, placebo-controlled phase 3 trial. Lancet doi:10.1016/ S0140-6736(13)62106-6 